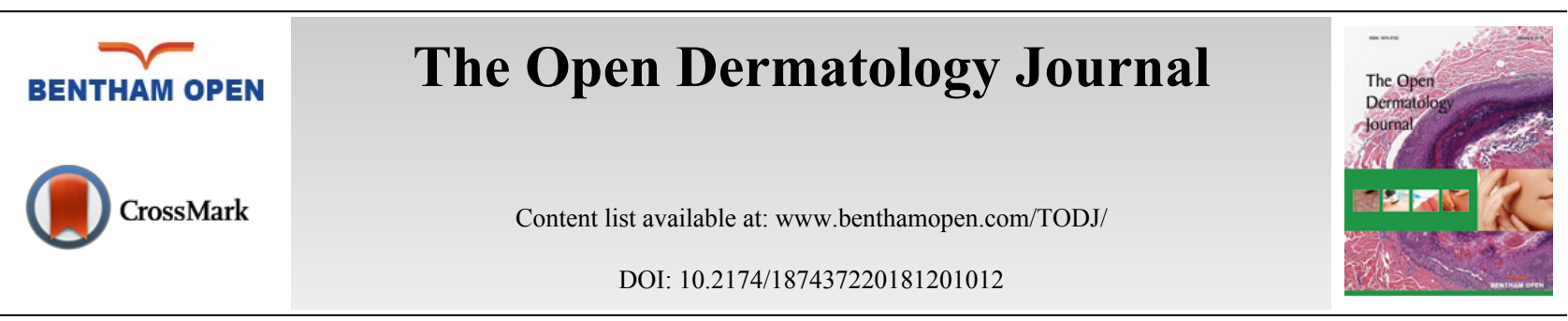

EDITORIAL

\title{
Patient Informational Sheet on Why Some Physicians are Hesitant to Prescribe Biologics
}

Biologics are a range of pharmaceutical products that interfere with specific parts of the immune system. In dermatology, most biologics are of receptor constructs that eliminate certain proteins involved with the normal body protective forces. These drugs have merit when treating psoriatic arthritis and erythrodermic, pustular psoriasis, but there are alarming concerns that trouble me when treating patients with limited psoriasis. And there is little doubt that pharmaceutical companies are advertising their biologic therapies to patients with minimal psoriasis, who in turn are expecting to be treated with these agents.

So first, are biologics as good as advertised on the media. I suggest they are not. Indeed, most pharmaceutical studies with these agents seek an end point of only $75 \%$ reduction in body coverage and severity. If this is their study results, let their advertising also reflect these less than glorious results.

Secondly, are biologics safe? I would suggest that any product that has a box warning stating that the product has been associated with serious infections and malignancies, are not free of side effects. In truth, the FDA and indeed the pharmaceutical company themselves, are saying that these side effects are real by providing information on the drug leaflet.

A third parameter might be, are these drugs expensive. I would say the cost borders on ridiculous. The wholesale acquisition cost of biologics can exceed \$15,000 per month. Assuming a patient acquires psoriasis at age ten and lives to 80 , then the cost of biologic therapy for her psoriasis would be close to $\$ 14,000,000$, using present costs. This does not include physician visits nor any topical therapy that might be added to psoriasis therapy. And of course, this amount would not include care for other skin problems or other medical and surgical needs unrelated to the psoriasis. If one believes that health care professionals have to give care in a fiscally responsive manner, this makes little sense.

The last issue that I raise relates to prior authorization approval that needs to accompany patients who request these drugs. These forms require physicians to sign that the medication is medically necessary and that the patient was a failure when treated with methotrexate. My major point would be that very few patients do not respond to methotrexate. A physician may prefer the side effect profile of biologics over methotrexate, but that's not what a doctor is attesting to on the prior authorization form. In short, the physician normally has to lie and state that the patient did not respond to methotrexate. I am not sure that these inaccuracies may not come back to haunt these physicians in the future.

I have recently put my thoughts into an informational sheet (see below) for my patients to read. Truly, medicine is the interaction between physician and patient. In this era, where the media is on a large scale providing drug information it is this one-on-one relationship in which physicians should discuss his/her concerns and thoughts related to psoriasis treatment. This patient handout below is how I started discussing biologics with patients. I welcome your feedback.

Patient informational sheet

Craig G. Burkhart, MPH, MD

5600 Monroe Street, Suite 106B, Sylvania, Ohio 43560

Information on why some physicians are hesitant to prescribe biologics (such as Enbrel, Humira, Taltz, Stelara, Remicade, Cimzia).

Biologics are actually a range of pharmaceutical products that interfere with specific parts of the immune system. In 
dermatology, most biologics are of receptor constructs that eliminate specific proteins involved in the normal immune response. Because these drugs interfere with the normal body immune response, they are associated with side effects, such as serious bacterial infections and malignancies as stated in their package inserts (boxed warnings stressing the significance of concern).

To clarify, biology is a natural science concerned with the study of life and living organisms. There is very little association between biology and biologics except they both sound scientific and positive.

Biologics are expensive. A study looking at the wholesale acquisition cost of some biologics was as high as $\$ 15,000$ per month [1]. Also, it should be kept in mind that psoriasis is a chronic disease, and so these costs compound over the duration of therapy. In this era of co-pay reduction cards, the pharmaceutical companies hope that we are simplistic enough to believe that the cost for their drugs are low because the patients' co-pays are low. Even if patients don't pay the high cost at the pharmacy, their insurance premiums will be raised to compensate for the cost. Also, eventually the costs also have to be shouldered by society at large. One of my tenets is a health care professional, I have to provide care in a fiscally responsive manner.

Biologics help, but rarely cure psoriasis. Indeed, most studies performed are for an end point of $75 \%$ reduction in psoriasis area on body and severity. Please note that it is not $100 \%$. I do wish to note that some patients may find these drugs life changing, especially those with psoriatic arthritis.

The media blitz of all the rewards of skin psoriasis from biologics may be overstated in commercials, but they have certainly increased patients' expectations. Ten years earlier, this suggesting to patients that they should wear long pants in order to hide lesions on the legs was acceptable.. Today, many patients don't want to look in the mirror and see any psoriasis.

Because of the expense of biologics, health insurances require all patients to first try oral methotrexate for their psoriasis. The technical terms would be that physicians must seek prior authorization for patients to receive biologics; prior authorization means that the treatment is medically necessary; and the physician must verify that such patients failed to respond to methotrexate prior to asking health insurances to pay for biologics. Methotrexate is an antimetabolite (it blocks folinic acid) and is one of the standard medications known as anti-cancer drug. This drug is used because almost $100 \%$ of psoriasis patients respond to methotrexate. It works even better than biologics, but it has an even higher propensity for serious side effects, especially the liver toxicity. I ask health insurance companies to pay for biologics, for those who don't respond to methotrexate, The scenario is that the doctor has to lie to the health insurance agencies for the patient to get biologics, and I refuse to lie to the health insurance agencies.

\section{REFERENCE}

[1] D'Souza LS, Payette MJ. Estimated cost and efficacy of systemic treatments that are approved by the US Food and Drug Administration for treatment of moderate to severe psoriasis. J Am Acad Dermatol 2015;72:589-598

Craig G Burkhart

5600 Monroe Street

Suite 106B

Sylvania

Ohio

University of Toledo

Department of Medicine

Toledo

Ohio

Ohio University

Department of Osteopathic Medicine

Athens

Ohio 